04

\title{
Эллипсоидальная модель для малых многослойных частиц
}

\author{
(C) В.Г. Фарафонов ${ }^{1}$, В.И. Устимов ${ }^{1}$, В.Б. Ильин ${ }^{1-3}$, М.В. Соколовская ${ }^{1}$ \\ ${ }^{1}$ Государственный университет аэрокосмического приборостроения, \\ 190000 Санкт-Петербург, Россия \\ ${ }^{2}$ Санкт-Петербургский государственный университет, \\ 199034 Санкт-Петербург, Россия \\ ${ }^{3}$ Главная (Пулковская) астрономическая обсерватория РАН, \\ 196140 Санкт-Петербург, Россия \\ e-mail: far@aanet.ru
}

Поступила в редакцию 22.09.2017г.

В настоящей работе построена эллипсоидальная модель для малых слоистых несферических частиц, предложены способы построения „эффективных“ многослойных эллипсоидов, светорассеивающие свойства которых были бы близки к свойствам исходных частиц. Для осесимметричных частиц речь идет о вытянутых или сплюснутых сфероидах (эллипсоидах вращения). Численные расчеты поляризуемости и сечений рассеяния малых слоистых несферических частиц, в том числе неконфокальных (подобных) сфероидов, чебышевских частиц и псевдосфероидов, проводятся разными приближенными и строгими методами. К приближенным подходам относится использование эллипсоидальной модели, в рамках которой поляризуемость слоистой частицы определяется двумя способами. В первом случае она вычисляется в приближении конфокальных сфероидов (CEA), а во втором - как линейная комбинация поляризуемостей вложенных сфероидов пропорционально объемам слоев (LVA). Из строгих методов применяются ЕВCM (метод расширенных граничных условий) и обобщенный SVM (метод разделения переменных). На основании сравнения результатов, полученных с помощью строгих и приближенных подходов, обсуждены недостатки и достоинства последних.

DOI: $10.21883 /$ OS.2018.02.45531.187-17

\section{1. Введение}

Рассеяние света малыми однородными и слоистыми частицами, размеры которых существенно меньше длины волны излучения, представляет большой интерес в различных областях науки $[1,2,3]$ и в особенности в нанооптике [4]. В этом случае наиболее часто используется приближение Релея [1,5].

Данный подход базируется на решении электростатической задачи, в рамках которой частица находится в постоянном внешнем электрическом поле и определяется „рассеянное“ поле. Это поле рассматривается в дальней зоне, т.е. достаточно далеко от рассеивателя, при этом следует учитывать только главный член, соответствующий дипольной составляющей. Таким образом, малая частица в релеевском приближении рассеивает электромагнитное излучение как некий диполь, момент которого напрямую связан с поляризуемостью частицы. Тензор поляризуемости рассматриваемой малой частицы полностью характеризует ее светорассеивающие свойства.

Изначально лорд Релей использовал приближение для однородных эллипсоидальных частиц [6]. Он отмечал, что при условии $d \ll \lambda$ и $|m| d \ll \lambda$, где $d$ и $m-$ наибольшая протяженность и показатель преломления частицы соответственно, $\lambda$ - длина волны падающего на частицу излучения, оптические свойства могут быть легко выражены через поляризуемость $\alpha$, причем для малых эллипсоидальных частиц выражение для $\alpha$ включает лишь одномерный интеграл, а для сфероидальных частиц оно может быть найдено аналитически [2]. Недавние уточнения приближения Релея для однородных эллипсоидов приводятся, например, в [7]. Приближение было распространено на ансамбли шаров [8,9], а также сформулировано для заряженных шаров $[10,11]$ и ансамблей заряженных шаров [12].

Приближение Релея было распространено на слоистые эллипсоиды. В работе [13] было построено в явном виде точное решение электростатической задачи для многослойных конфокальных эллипсоидов в результате обобщения двуслойного случая, рассмотренного в монографии [1]. Позже была предпринята попытка применения данного подхода для многослойных неконфокальных эллипсоидов [14] посредством разбиения слоев на большое количество тонких подслоев, поверхности которых постепенно менялись от внутренней к внешней границе слоя. Однако численное моделирование показало, что это достаточно громоздкое приближенное решение имеет узкую область применимости [15].

Для небольших неэллипсоидальных рассеивателей поляризуемость $\alpha$ может быть найдена численно из решения некоторого поверхностного интегрального уравнения. Это было сделано для нескольких форм частиц: конечных цилиндров, конусов и параллелепипедов (см., например, $[2,16])$. Итеративный способ получения $\alpha$ для небольшой частицы произвольной формы был разработан А. Раммом (см. [17] и ссылки, данные в этой монографии). Однако оба способа являются достаточно сложными вычислительно. 
Еще одним общим подходом является решение электростатической задачи методами, используемыми для решение задачи рассеяния света для слоистых частиц. Такие методы включают в первую очередь метод разделения переменных (SVM) со сферическим базисом, который обычно применялся при рассмотрении рассеяния света слоистыми шарами $[18,19]$. Это SVM-решение сравнительно просто, но во многих приложениях форма частиц не может быть проигнорирована. Метод SVM со сфероидальным базисом был применен сначала к двуслойным сфероидам [20], а затем и к многослойным [21]. Однако требуемое при этом вычисление сфероидальных функций требует значительных затрат, а кроме этого, таким образом могут быть рассмотрены лишь частицы, имеющие сфероидальные слои с софокусными границами.

Слоистые рассеиватели произвольной (обычно осесимметричной) формы исследовались методом расширенных граничных условий (ЕВСМ). Классическими работами здесь является теоретическая статья [22] и публикация [23] с численными результатами. Многие работы, которые применяли такой подход к слоистым рассеивателям, процитированы во всеобъемлющих обзоpax $[24,25]$. Еще два подобных метода - обобщенный метод поточечной сшивки (РММ) и метод обобщенных мультиполей (GMT) применялись к слоистым частицам, например в [26] и [27] соответственно. Другие методы метод связных диполей (coupled dipole method, CDM), метод моментов (method of moments, MoM), метод конечных разностей (finite difference time domain method, FDTD) и др. $[28,29]$ - являются более универсальными и поэтому требующими существенно большего времени вычислений, чем методы SVM, EBCM, PMM и GMT, даже при применении к слоистым частицам простой формы.

В общем случае приближение Релея на основе строгого решения электростатической задачи для малых многослойных частиц было построено в работе [30]. Здесь был разработан подход, аналогичный методу расширенных граничных условий (ЕВСМ), область применимости которого имеет существенные ограничения [31], в том числе для конфокальных и неконфокальных слоистых сфероидов [32]. В связи с этим обстоятельством представляется важным построение достаточно простого приближенного решения задачи рассеяния света малыми слоистыми частицами с широкой областью применимости. Примером удачного решения подобной задачи для малых однородных несферических частиц может служить элиппсоидальная модель [33]. В ее рамках исходная частица заменяется ,эквивалентным“ эллипсоидом, при этом их рассеивающие свойства должны быть достаточно близки.

В настоящей работе предлагается эллипсоидальная модель для малых слоистых частиц. Сначала рассматривается приближение Релея для многослойных конфокальных эллипсоидов и сфероидов. Далее обсуждаются способы построения „эквивалентных“ слоистых эллипсоидов и сфероидов для несферических частиц разных видов - параллелепипедов, псевдосфероидов и чебышевских частиц. Последний раздел посвящен анализу результатов численных расчетов, полученных с помощью строгих методов решения задачи для рассматриваемых частиц (EBCM, SVM) и в рамках предлагаемой эллипсоидальной модели. Завершают работу выводы.

\section{Релеевское приближение для многослойных эллипсоидов}

Поверхность однородной эллипсоидальной частицы с центром в начале координат декартовой системы $(x, y, z)$ задается уравнением

$$
\frac{x^{2}}{a^{2}}+\frac{y^{2}}{b^{2}}+\frac{z^{2}}{c^{2}}=1
$$

где $a, b, c$ - полуоси эллипсоида. Для описания конфокальных частиц целесообразно ввести эллипсоидальную систему координат $(\xi, \eta, \xi)$, которая связана с декартовой системой следующим образом:

$$
\begin{gathered}
\frac{x^{2}}{a^{2}+\xi}+\frac{y^{2}}{b^{2}+\xi}+\frac{z^{2}}{c^{2}+\xi}=1, \quad-c^{2}<\xi<\infty, \\
\frac{x^{2}}{a^{2}+\eta}+\frac{y^{2}}{b^{2}+\eta}+\frac{z^{2}}{c^{2}+\eta}=1, \quad-b^{2}<\eta<-c^{2}, \\
\frac{x^{2}}{a^{2}+\xi}+\frac{y^{2}}{b^{2}+\xi}+\frac{z^{2}}{c^{2}+\xi}=1, \quad-a^{2}<\xi<-b^{2},
\end{gathered}
$$

где предполагается, что $a>b>c-$ полуоси ядра частицы. Из соотношений (2) нетрудно найти зависимость декартовых координат от эллиптических:

$$
\begin{aligned}
& x^{2}=\frac{\left(a^{2}+\xi\right)\left(a^{2}+\eta\right)\left(a^{2}+\xi\right)}{\left(a^{2}-b^{2}\right)\left(a^{2}-c^{2}\right)}, \\
& y^{2}=\frac{\left(b^{2}+\xi\right)\left(b^{2}+\eta\right)\left(b^{2}+\xi\right)}{\left(b^{2}-a^{2}\right)\left(b^{2}-c^{2}\right)}, \\
& z^{2}=\frac{\left(c^{2}+\xi\right)\left(c^{2}+\eta\right)\left(c^{2}+\xi\right)}{\left(c^{2}-a^{2}\right)\left(c^{2}-b^{2}\right)} .
\end{aligned}
$$

Отметим, что правые части в приведенных выше формулах всегда положительны. Это связано с тем, что количество отрицательных множителей в числителях и знаменателях дробей одинаково (0, 1 и 2 соответственно).

Эллипсоиды называются конфокальными, если их поверхности являются координатными в единой эллипсоидальной системе координат, т. е. их поверхности должны описываться первым уравнением из соотношений (2) при фиксированной первой координате:

$$
\frac{x^{2}}{a^{2}+\xi_{j}}+\frac{y^{2}}{b^{2}+\xi_{j}}+\frac{z^{2}}{c^{2}+\xi_{j}}=1 .
$$

Будем нумеровать поверхности слоев конфокального эллипсоида от внешней оболочки $-\xi_{1}>\xi_{2}>\ldots>\xi_{J}=0$, где $J-$ количество слоев. Таким образом, условие 
конфокальности для многослойного эллипсоида может быть записано следующим образом:

$$
a_{1}^{2}+\xi_{j}=a_{j}^{2}, \quad b_{1}^{2}+\xi_{j}=b_{j}^{2}, \quad c_{1}^{2}+\xi_{j}=c_{j}^{2},
$$

где $j=1,2, \ldots, J$. Иначе данное условие можно записать в виде

$$
a_{j}^{2}-b_{j}^{2}=d_{1}^{2}, \quad a_{j}^{2}-c_{j}^{2}=d_{2}^{2},
$$

где $j=1,2, \ldots, J$, при этом $d_{1}, d_{2}-$ половина фокусных расстояний эллипсов, являющихся сечениями эллипсоида координатными плоскостями $z=0$ и $y=0$, при этом $d_{1}>d_{2}$.

Рассеяние электромагнитного излучения частицами малыми относительно длины волны можно рассматривать в приближении Релея, которое базируется на понятии поляризуемости частицы $\alpha$. По определению она связывает напряженность внешнего постоянного поля $\mathbf{E}_{0}$ с дипольным моментом $\mathbf{p}$,рассеянного“ поля в дальней зоне, которое образуется вследствие присутствия частицы:

$$
\mathbf{p}=\alpha \mathbf{E}_{0} .
$$

Из этого определения следует, что поляризуемость $\alpha$ представляет собой тензор, который для эллипсоидов, параллелепипедов [34] и осесимметричных частиц [35] в том числе многослойных является диагональным.

Релеевское приближение для однородных эллипсоидов является классической задачей, решение которой хорошо известно $[1,5]$. Сечения поглощения и рассеяния зависят от волнового числа $k$ и поляризуемости частицы:

$$
\begin{gathered}
C^{\mathrm{abs}}=k \operatorname{Im}\left(l^{2} \alpha_{x}+m^{2} \alpha_{y}+n^{2} \alpha_{z}\right), \\
C^{\text {sca }}=\frac{k^{4}}{6 \pi}|\alpha|^{2},
\end{gathered}
$$

где

$$
|\alpha|^{2}=l^{2}\left|\alpha_{x}\right|^{2}+m^{2}\left|\alpha_{y}\right|^{2}+n^{2}\left|\alpha_{z}\right|^{2},
$$

$l, m, n-$ направляющие косинусы напряженности внешнего электрического поля $\mathbf{E}_{0}$ относительно трех главных осей тензора поляризуемости (они же оси декартовой системы). Например, в случае ориентации внешнего поля вдоль оси $z$ поляризуемость эллипсоида с полуосями $a, b, c$ вычисляется по формуле

$$
\alpha_{z}=\frac{4 \pi}{3} a b c \frac{(\varepsilon-1)}{1+(\varepsilon-1) L_{z}},
$$

где $\varepsilon-$ относительная диэлектрическая проницаемость среды внутри частицы. Для геометрического фактора $L_{z}$, зависящего только от формы эллипсоида, имеем

$$
L_{z}=\frac{a b c}{2} \int_{0}^{\infty} \frac{\mathrm{d} q}{\left(c^{2}+q\right) f(q)}
$$

где $f(q)=\sqrt{\left(a^{2}+q\right)\left(b^{2}+q\right)\left(c^{2}+q\right)}$. Для других факторов $L_{x}$ и $L_{y}$ в формулах (11), (12) нужно поменять лишь полуоси, а именно, $c^{2}$ следует заменить на $a^{2}$ и $b^{2}$ соответственно.

Поляризуемость частицы связана с $T$-матрицей и определяется следующим образом [34]:

$$
\alpha_{z}=\frac{4 \pi}{3} T_{11}
$$

где $T_{11}$ - первый элемент $T$-матрицы. В рассматриваемом случае $T$-матрица представляет собой число и записывается следующим образом [36]:

$$
T=T_{11}=-\frac{A_{2}}{A_{1}}
$$

где числа $A_{1}$ и $A_{2}$ находятся из матричного уравнения

$$
\begin{gathered}
\left(\begin{array}{l}
A_{1} \\
A_{2}
\end{array}\right)=\left(\begin{array}{cc}
{\left[1+\left(\varepsilon_{2}-1\right) L_{z}^{1}\right]} & \left(\varepsilon_{2}-1\right) \frac{L_{z}^{1}\left(L_{z}^{1}-1\right)}{\tilde{V}_{1}} \\
-\left(\varepsilon_{2}-1\right) \tilde{V}_{1} & {\left[1-\left(\varepsilon_{2}-1\right)\left(L_{z}^{1}-1\right)\right]}
\end{array}\right) \cdots \\
\ldots\left(\begin{array}{cc}
{\left[1+\left(\varepsilon_{J}-1\right) L_{z}^{J-1}\right]} & \left(\varepsilon_{J}-1\right) \frac{L_{z}^{J-1}\left(L_{z}^{J-1}-1\right)}{\tilde{V}_{J-1}} \\
-\left(\varepsilon_{J}-1\right) \tilde{V}_{J-1} & -\left[1-\left(\varepsilon_{J}-1\right)\left(L_{z}^{J-1}-1\right)\right]
\end{array}\right) \\
\times\left(\begin{array}{c}
{\left[1+\left(\varepsilon_{J+1}-1\right) L_{z}^{J}\right]} \\
-\left(\varepsilon_{J+1}-1\right) \tilde{V}_{J}
\end{array}\right) .
\end{gathered}
$$

Здесь $\varepsilon_{j+1}=\tilde{\varepsilon}_{j+1} / \tilde{\varepsilon}_{j}$ - относительные диэлектрические проницаемости, а факторы $\tilde{V}_{j}=a_{j} b_{j} c_{j}$, т.е. связаны с объемами соответствующих внутренних эллипсоидов, при $j=1$ получим объем частицы. В случае горизонтальной ориентации внешнего поля поляризуемость частицы вычисляется аналогично с точностью до замены факторов $L_{z}^{j}$ на факторы $L_{x}^{j}$.

Ранее [13] для многослойного конфокального эллипсоида электростатическая задача была решена иначе без привлечения $T$-матрицы, при этом поляризуемость частицы представлялась в виде

$$
\alpha_{z}=\frac{4 \pi}{3} \frac{\left(\tilde{A}_{2}-\tilde{A}_{1}\right) \tilde{V}_{1}}{\tilde{A}_{1}+\left(\tilde{A}_{2}-\tilde{A}_{1}\right) L_{z}^{(1)}}
$$

где для чисел $\tilde{A}_{1}$ и $\tilde{A}_{2}$ справедливо уравнение

$$
\left(\begin{array}{c}
\tilde{A}_{1} \\
\tilde{A}_{2}
\end{array}\right)=\left(\begin{array}{cc}
\tilde{V}_{1} & L_{z}^{1} \\
\varepsilon_{2} \tilde{V}_{1} & \varepsilon_{2}\left(L_{z}^{1}-1\right)
\end{array}\right) \Psi,
$$

при этом матрица $\Psi$ представляет собой правую часть уравнения (15) без первого матричного множителя. Для доказательства тождественности этих результатов достаточно провести следующие вычисления:

$$
\left(\begin{array}{c}
\left(\tilde{A}_{2}-\tilde{A}_{1}\right) \tilde{V}_{1} \\
\tilde{A}_{1}+\left(\tilde{A}_{2}-\tilde{A}_{1}\right) L_{z}^{(1)}
\end{array}\right)=\left(\begin{array}{c}
-A_{2} \tilde{V}_{1} \\
A_{1} \tilde{V}_{1}
\end{array}\right)=\left(\begin{array}{c}
-A_{2} \\
A_{1}
\end{array}\right),
$$

из которых эквивалентность соотношений 
Второй подход полезен для определения „эффективной“ диэлектрической проницаемости многослойного конфокального эллипсоида $\varepsilon_{\mathrm{ef}}$. Сравнивая формулы (11) и (16) для однородного и многослойного конфокального эллипсоидов, нетрудно сделать вывод, что первый с „эффективной“ диэлектрической проницаемостью

$$
\varepsilon_{\mathrm{ef}}=\frac{\tilde{A}_{2}}{\tilde{A}_{1}}
$$

будет иметь точно такую же поляризуемость как и второй, т. е. обе частицы будут рассеивать свет одинаково.

В частном случае сфероидов две полуоси одинаковы, при этом уравнение (1) принимает вид

$$
\frac{x^{2}+y^{2}}{b^{2}}+\frac{z^{2}}{a^{2}}=1
$$

для вытянутых и

$$
\frac{x^{2}+y^{2}}{a^{2}}+\frac{z^{2}}{b^{2}}=1
$$

для сплюснутых частиц, при этом всегда $a>b$. Соотношения (2), (3) использовать нельзя, поэтому используется вытянутая или сплюснутая система координат $(\xi, \eta, \varphi)$, где $\varphi$ - азимутальный угол. Электростатическая задача решается в сфероидальной системе координат [36], при этом геометрические факторы (12) могут быть записаны в виде

$$
\begin{gathered}
L_{z}=\left(\xi^{2}-1\right)\left(\frac{\xi}{2} \ln \frac{\xi+1}{\xi-1}-1\right), \\
\xi=(a / b)\left((a / b)^{2}-1\right)^{-1 / 2}=\frac{a}{\sqrt{a^{2}-b^{2}}}
\end{gathered}
$$

для вытянутых и

$$
\begin{aligned}
& L_{z}=\left(\xi^{2}+1\right)\left(1-\xi \arctan \frac{1}{\xi}\right), \\
& \xi=\left((a / b)^{2}-1\right)^{-1 / 2}=\frac{b}{\sqrt{a^{2}-b^{2}}}
\end{aligned}
$$

для сплюснутых частиц соответственно. Для трех геометрических факторов справедливо условие

$$
L_{x}+L_{y}+L_{z}=1
$$

поэтому можно для двух других факторов использовать формулу $L_{x}=L_{y}=\left(1-L_{z}\right) / 2$.

Для многослойных сфероидов вместо уравнения (4) следует писать уравнения аналогичные (20),(21), при этом для конфокальных частиц вместо соотношений $(5),(6)$ следует требовать выполнения условий

$$
a_{1}^{2}-b_{1}^{2}=a_{2}^{2}-b_{2}^{2}=\ldots=a_{J}^{2}-b_{J}^{2}=\left(\frac{d}{2}\right)^{2},
$$

где $d-$ половина фокусного расстояния. Отметим, что в рассматриваемом случае фокусы поверхностей оболочек совпадают, а сами поверхности являются координатными в единой сфероидальной системе координат.

Поляризуемость многослойных конфокальных сфероидальных частиц вычисляется по формулам (13) или (16), при этом геометрические факторы нужно определять по соотношениям (22), (23).

Аналитические формулы для поляризуемости других частиц с простой формой - конечных цилиндров, конусов, параллелепипедов и т.д. - отсутствуют, поскольку их поверхности не являются координатными в какихлибо ортогональных системах.

\section{Эллипсоидальная модель и способы вычисления поляризуемости}

Подход, связанный с применением эллипсоидальной модели для многослойной частицы, предполагает замену каждой поверхности слоев исходной частицы на эллипсоидальную поверхность. Ранее для однородной частицы оптимальный выбор модельного эллипсоида имел место при выполнении двух условий. Во-первых, объемы модельного эллипсоида и несферической частицы должны совпадать:

$$
V_{\text {ef }}=V_{\text {part }} \text {. }
$$

Во-вторых, должны быть одинаковыми отношения эффективных полуосей эллипсоида и отношения соответствующих размеров частицы:

$$
\frac{a_{\mathrm{ef}}}{c_{\mathrm{ef}}}=\frac{a_{\mathrm{part}}}{c_{\mathrm{part}}}, \quad \frac{b_{\mathrm{ef}}}{c_{\mathrm{ef}}}=\frac{b_{\mathrm{part}}}{c_{\mathrm{part}}} .
$$

Отметим, что в некоторых случаях (псевдосфероиды) можно фиксировать не отношения размеров, а сами размеры модельного эллипсоида и несферической частицы:

$$
a_{\text {ef }}=a_{\text {part }}, \quad c_{\text {ef }}=c_{\text {part }} .
$$

В случае осесимметричных частиц в соотношениях $(27),(28)$ достаточно оставить одно равенство. Теперь для слоистой частицы соотношения (26)-(28) являются необходимыми и достаточными для построения модельных эллипсоидов, соответствующих внутренним областям для каждой из поверхностей слоев.

Сначала рассмотрим прямоугольный параллелепипед со сторонами $a, b, c$ вдоль осей декартовой системы координат $x, y, z$. При этом предполагается, что начало координат совмещено с центром параллелепипеда. Для определения полуосей $a_{\mathrm{ef}}, b_{\mathrm{ef}}$ и $c_{\mathrm{ef}}$ модельного эллипсоида в этом случае следует воспользоваться соотношениями (26), (27). В результате получим

$$
a_{\mathrm{ef}}=\sqrt[3]{\frac{3}{4 \pi}} a, \quad b_{\mathrm{ef}}=\sqrt[3]{\frac{3}{4 \pi}} b, \quad c_{\mathrm{ef}}=\sqrt[3]{\frac{3}{4 \pi}} c .
$$

Применение формулы (28) для параллелепипеда нецелесообразно, так как в этом случае получим изменение размера эффективного эллипсоида только в направлении оси $y\left(b_{\mathrm{ef}}=3 / 4 \pi b\right)$, чему нет разумного объяснения. 
Теперь обратимся к осесимметричным частицам. В этом случае „эффективный“ эллипсоид превращается в вытянутый или сплюснутый „эффективный“ сфероид, а при равенстве продольных и поперечных размеров в „эффективный“ шар. Для определения полуосей $a_{\mathrm{ef}}, b_{\mathrm{ef}}$ $\left(a_{\mathrm{ef}}=b_{\mathrm{ef}}\right)$ и $c_{\text {ef }}$ следует воспользоваться соотношением (26) и одним из уравнений (27):

$$
\frac{a_{\mathrm{ef}}}{c_{\mathrm{ef}}}=\frac{a_{\mathrm{part}}}{c_{\mathrm{part}}}
$$

так как для определения двух полуосей достаточно двух уравнений.

Для чебышевских частиц уравнение поверхности записывается следующим образом:

$$
r=a(1+\epsilon \cos n \theta)
$$

где $n$ определяет количество максимумов на поверхности, $a$ - радиус невозмущенного шара, при этом параметр возмущения $|\epsilon|<1$. Отметим, что ниже будет рассматриваться только улитка Паскаля, для которой $n=1$ и объем $V=4 \pi / 3 a^{3}\left(1+\epsilon^{2}\right)$.

В данном случае решение уравнений (26), (27) дает „эффективный“ сплюснутый сфероид с полуосями

$$
a_{\mathrm{ef}}=b_{\mathrm{ef}}=\sqrt[3]{\frac{d}{h}\left(1+\epsilon^{2}\right)} a, \quad c_{\mathrm{ef}}=\sqrt[3]{\frac{h^{2}}{d^{2}}\left(1+\epsilon^{2}\right)} a
$$

где $d$ и $h$ - ширина и высота улитки Паскаля (31). Эти характеристики нужно определять как максимумы функций $r(\theta) \sin \theta$ и $r(\theta) \cos \theta$ соответственно. В результате для ширины получим

$$
d=\frac{1}{2 \sqrt{2}} \frac{(t+3)^{3 / 2}}{(t+1)^{1 / 2}} a,
$$

где $t=\sqrt{1+8 \epsilon^{2}}$. Высота $h=2 a$ для выпуклых частиц, т. е. при $\epsilon<0.5$, и

$$
h=\frac{(2 \epsilon+1)^{2}}{4 \epsilon} a
$$

для невыпуклых, т.е. при достаточно большом параметре возмущения: $0.5 \leq \epsilon \leq 1.0$.

В заключение рассмотрим сплюснутые псевдосфероиды:

$$
r(\theta)=a \sqrt{1-\epsilon^{2} \cos ^{2} \theta}, \quad \epsilon^{2}=1-b^{2} / a^{2} \geq 0
$$

с полуосями $a=1$ и $b \leq 1$. Данные частицы получаются в результате инверсии относительно начала координат вытянутых сфероидов $r(\theta)=b / \sqrt{1-\epsilon^{2} \cos ^{2} \theta}$ с теми же полуосями и фокусным расстоянием $d=2 \sqrt{a^{2}-b^{2}}$. Вычисление объема псевдосфероида дает

$$
V=\frac{2 \pi}{3} a^{3} \int_{-1}^{1}\left(1-\epsilon^{2} x^{2}\right)^{3 / 2} d x
$$

В данном случае для построения модельного сфероида целесообразно фиксировать ширину частицы [33]:

$$
a_{\mathrm{ef}}=b_{\mathrm{ef}}=a, \quad c_{\mathrm{ef}}=\frac{3 V}{4 \pi a^{2}},
$$

при этом последняя полуось $c_{\text {ef }}$ вычисляется из требования равенства объемов (26).

Приведенные выше процедуры позволяют заменить исходные частицы „эффективными“ эллипсоидами или сфероидами. Поляризуемость „эффективных“ частиц предлагается определять двумя способами. В первом случае будем использовать СЕА - приближение конфокальных эллипсоидов (13)-(15), во втором - LVA, т. е. аппроксимацию, соответствующую линейной комбинации поляризуемостей соответствующих слоев:

$$
\begin{gathered}
\alpha_{z}=\alpha_{1} \frac{V_{1}-V_{2}}{V_{1}}+\alpha_{2} \frac{V_{2}-V_{3}}{V_{1}}+\ldots \\
+\alpha_{J-1} \frac{V_{J-1}-V_{J}}{V_{1}}+\alpha_{J} \frac{V_{J}}{V_{1}},
\end{gathered}
$$

где $V_{1}$ - объем частицы, $V_{2}, V_{3}, \ldots, V_{J}$ - объемы областей внутри поверхностей, начиная с внешней и заканчивая ядром. Поляризуемости $\alpha_{1}, \alpha_{2}, \ldots, \alpha_{J}$ вычисляются для однородных „эффективных“ эллипсоидов или сфероидов, соответствующих поверхностям слоев, т.е. начиная от всей частицы до ядра включительно. Заметим, что приближение (38) для поляризуемости многослойной частицы не учитывает влияние слоев частицы друг на друга.

\section{Результаты численных расчетов и обсуждение}

Численные расчеты проводились для конфокальных и неконфокальных сфероидов, псевдосфероидов и улиток Паскаля (рис. 1). При этом использовались как приближения CEA и LVA, так и строгие методики методов ЕBCM и SVM. При этом в большинстве случаев конечными результатами служили сечения поглощения $C_{\text {abs }}$ и рассеяния $C_{\text {sca }}$ многослойных частиц.

Сначала будем сравнивать результаты расчетов поляризуемостей двухслойных конфокальных эллипсоидов на основании CEA и LVA. Первая аппроксимация в данном случае представляет точное решение, а вторая приближенное. Из рис. 2 видно, что относительная погрешность LVA менее $1 \%$ для реальной части и 2\% для мнимой. Из этих оценок следует, что вычисления сечений по формулам (8), (9) с помощью приближения LVA будут давать относительные погрешности менее $2 \%$. Отметим, что подобная близость данных по алгоритмам CEA и LVA достаточно удивительна, поскольку первый в отличии от второго нелинейно зависит от объемов слоев частицы. 

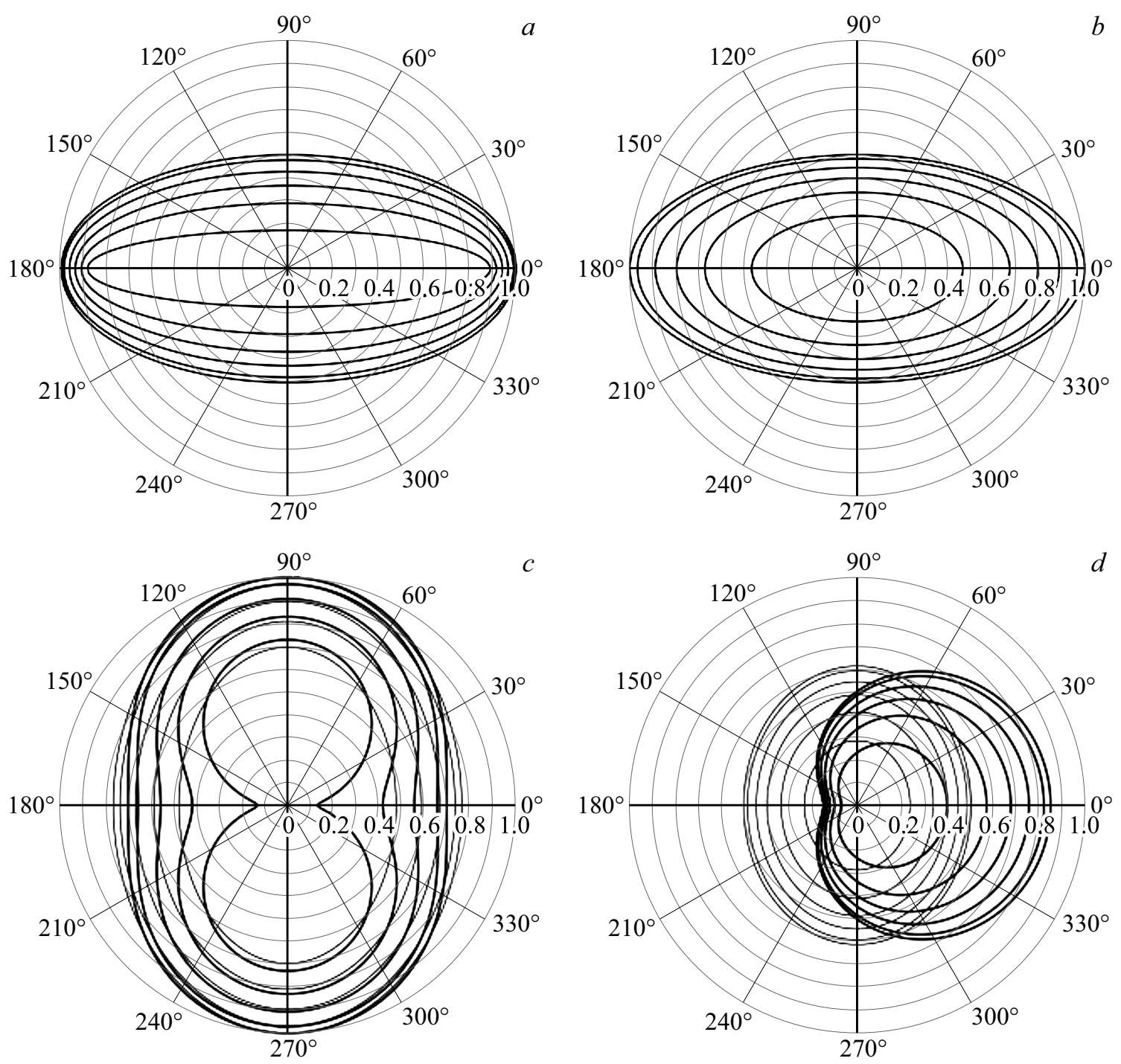

Рис. 1. Слоистые осесимметричные частицы разных видов (осевое сечение): $a$ - многослойный конфокальный сфероид $\left(a_{1} / b_{1}=2\right) ; b-$ неконфокальный сфероид с подобными слоями $\left(a_{j} / b_{j}=2\right) ; c$ - псевдосфероид со слоями, соответствующими конфокальным сфероидам с полуосями $a_{1}=1.0$ и $b_{1}=0.7$, „эффективные“ сплюснутые сфероиды для исходного и внутренних псевдосфероидов представлены на рисунке тонкими линиями; $d-$ улитка Паскаля с подобными слоями, соответствующими параметру $\epsilon=0.7$, тонкие линии — „эффективные“ сплюснутые сфероиды для исходной и внутренних улиток.
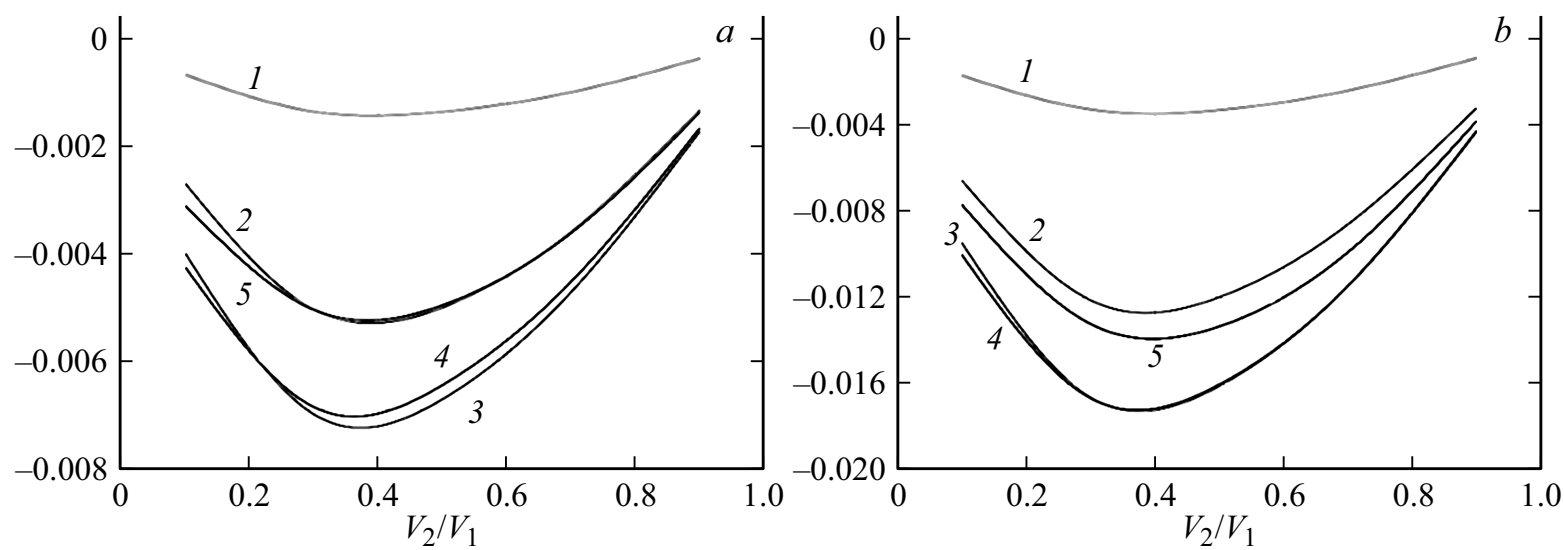

Рис. 2. Относительная погрешность поляризуемости $\left(\alpha_{\mathrm{LVA}}-\alpha_{\mathrm{CEA}}\right) / \alpha_{\mathrm{CEA}}$, рассчитанная по приближенной формуле (38) и точному алгоритму для двухслойных конфокальных сфероидов в зависимости от отношений объема ядра к объему частицы $V_{2} / V_{1}$. На рисунках кривые $1-5$ соответствуют случаям полуосей $a_{1}=1$ и $b_{1}=0.9,0.7,0.5,0.3,0.1$. $a$ - относительная погрешность действительных частей поляризуемостей; $b-$ мнимых частей. 
Таблица 1. Результаты расчета сечений $(k=0.5)$ трехслойных подобных сфероидов с диэлектрическими проницаемостями внешней оболочки $\tilde{\varepsilon}_{1}=1.69+0.10 j$, внутренней оболочки $\tilde{\varepsilon}_{2}=2.25+0.15 j$ и ядра $\tilde{\varepsilon}_{3}=2.89+0.20 j$

\begin{tabular}{|c|c|c|c|c|c|c|c|}
\hline \multirow{2}{*}{ Случай } & \multirow{2}{*}{$\begin{array}{c}\text { Параметры } \\
\text { сфероида }\end{array}$} & \multicolumn{3}{|c|}{ № оболочки $(i)$} & \multirow{2}{*}{$\begin{array}{c}\text { Метод } \\
\text { расчета }\end{array}$} & \multirow{2}{*}{$\begin{array}{c}\text { Сечение } \\
\text { рассеяния }\end{array}$} & \multirow{2}{*}{$\begin{array}{c}\text { Сечение } \\
\text { поглощения }\end{array}$} \\
\hline & & 1 & 2 & 3 & & & \\
\hline 1 & $\begin{array}{c}a \\
b \\
a / b \\
V[i]\end{array}$ & $\begin{array}{c}1.00 \\
0.80 \\
0.64000\end{array}$ & $\begin{array}{c}0.80 \\
0.64 \\
1.25 \\
0.32768\end{array}$ & $\begin{array}{c}0.60 \\
0.48 \\
0.13824\end{array}$ & $\begin{array}{c}\text { CEA } \\
\text { LVA } \\
\operatorname{EBCM}(16) \\
\operatorname{SVM}(16)\end{array}$ & $\begin{array}{l}1.68104 \mathrm{E}-02 \\
1.64991 \mathrm{E}-02 \\
1.68074 \mathrm{E}-02 \\
1.68074 \mathrm{E}-02\end{array}$ & $\begin{array}{l}1.05594 \mathrm{E}-01 \\
1.03978 \mathrm{E}-01 \\
1.05579 \mathrm{E}-01 \\
1.05579 \mathrm{E}-01\end{array}$ \\
\hline 2 & $\begin{array}{c}a \\
b \\
a / b \\
V[i]\end{array}$ & $\begin{array}{c}1.00 \\
0.60 \\
0.36000\end{array}$ & $\begin{array}{c}0.80 \\
0.48 \\
1.67 \\
0.18432\end{array}$ & $\begin{array}{c}0.60 \\
0.36 \\
0.07776\end{array}$ & $\begin{array}{c}\text { CEA } \\
\text { LVA } \\
\operatorname{EBCM}(16) \\
\operatorname{SVM}(16)\end{array}$ & $\begin{array}{c}6.02212 \mathrm{E}-03 \\
5.91601 \mathrm{E}-03 \\
6.15 \mathrm{E}-03 \\
6.01727 \mathrm{E}-03\end{array}$ & $\begin{array}{c}6.67507 \mathrm{E}-02 \\
6.57325 \mathrm{E}-02 \\
6.84 \mathrm{E}-02 \\
6.67061 \mathrm{E}-02\end{array}$ \\
\hline 3 & $\begin{array}{c}a \\
b \\
a / b \\
V[i]\end{array}$ & $\begin{array}{c}1.00 \\
0.40 \\
0.16000\end{array}$ & $\begin{array}{c}0.80 \\
0.32 \\
2.50 \\
0.08192\end{array}$ & $\begin{array}{c}0.60 \\
0.24 \\
0.03456\end{array}$ & $\begin{array}{c}\text { CEA } \\
\text { LVA } \\
\operatorname{EBCM}(16) \\
\operatorname{SVM}(16)\end{array}$ & $\begin{array}{c}1.38340 \mathrm{E}-03 \\
1.36311 \mathrm{E}-03 \\
- \\
1.38 \mathrm{E}-03\end{array}$ & $\begin{array}{c}3.42036 \mathrm{E}-02 \\
3.37465 \mathrm{E}-02 \\
- \\
3.41 \mathrm{E}-02\end{array}$ \\
\hline 4 & $\begin{array}{c}a \\
b \\
a / b \\
V[i]\end{array}$ & $\begin{array}{c}1.00 \\
0.20 \\
0.04000\end{array}$ & $\begin{array}{c}0.80 \\
0.16 \\
5.00 \\
0.02048\end{array}$ & $\begin{array}{c}0.60 \\
0.12 \\
0.00864\end{array}$ & $\begin{array}{c}\text { CEA } \\
\text { LVA } \\
\text { EBCM }(16) \\
\text { SVM }(16)\end{array}$ & $\begin{array}{c}1.02888 \mathrm{E}-04 \\
1.02061 \mathrm{E}-04 \\
- \\
-\end{array}$ & $\begin{array}{c}1.00793 \mathrm{E}-02 \\
1.00016 \mathrm{E}-02 \\
- \\
-\end{array}$ \\
\hline
\end{tabular}

Таблица 2. То же, что в табл. 1, но для трехслойных подобных псевдосфероидов

\begin{tabular}{|c|c|c|c|c|c|c|c|}
\hline \multirow{2}{*}{ Случай } & \multirow{2}{*}{$\begin{array}{c}\text { Параметры } \\
\text { псевдосфероида }\end{array}$} & \multicolumn{3}{|c|}{ № оболочки $(i)$} & \multirow{2}{*}{$\begin{array}{c}\text { Метод } \\
\text { расчета }\end{array}$} & \multirow{2}{*}{$\begin{array}{c}\text { Сечение } \\
\text { рассеяния }\end{array}$} & \multirow{2}{*}{$\begin{array}{c}\text { Сечение } \\
\text { поглощения }\end{array}$} \\
\hline & & 1 & 2 & 3 & & & \\
\hline 1 & $\begin{array}{c}a \\
b \\
a / b \\
V[i]\end{array}$ & $\begin{array}{c}1.00 \\
0.80 \\
0.83019\end{array}$ & $\begin{array}{c}0.80 \\
0.64 \\
1.25 \\
0.42506\end{array}$ & $\begin{array}{c}0.60 \\
0.48 \\
0.17932\end{array}$ & $\begin{array}{c}\text { CEA } \\
\text { LVA } \\
\operatorname{EBCM}(16) \\
\operatorname{SVM}(16)\end{array}$ & $\begin{array}{l}2.34468 \mathrm{E}-02 \\
2.30287 \mathrm{E}-02 \\
2.32650 \mathrm{E}-02 \\
2.32650 \mathrm{E}-02\end{array}$ & $\begin{array}{l}1.14885 \mathrm{E}-01 \\
1.13322 \mathrm{E}-01 \\
1.14084 \mathrm{E}-01 \\
1.14084 \mathrm{E}-01\end{array}$ \\
\hline 2 & $\begin{array}{c}a \\
b \\
a / b \\
V[i]\end{array}$ & $\begin{array}{c}1.00 \\
0.60 \\
0.71367\end{array}$ & $\begin{array}{c}0.80 \\
0.48 \\
1.67 \\
0.36540\end{array}$ & $\begin{array}{c}0.60 \\
0.36 \\
0.15415\end{array}$ & $\begin{array}{c}\text { CEA } \\
\text { LVA } \\
\text { EBCM }(16) \\
\text { SVM }(16)\end{array}$ & $\begin{array}{l}1.61714 \mathrm{E}-02 \\
1.58949 \mathrm{E}-02 \\
1.58393 \mathrm{E}-02 \\
1.58387 \mathrm{E}-02\end{array}$ & $\begin{array}{l}9.25890 \mathrm{E}-02 \\
9.14206 \mathrm{E}-02 \\
9.10351 \mathrm{E}-02 \\
9.10235 \mathrm{E}-02\end{array}$ \\
\hline 3 & $\begin{array}{c}a \\
b \\
a / b \\
V[i]\end{array}$ & $\begin{array}{c}1.00 \\
0.40 \\
0.64033\end{array}$ & $\begin{array}{c}0.80 \\
0.32 \\
2.50 \\
0.32785\end{array}$ & $\begin{array}{c}0.60 \\
0.24 \\
0.13831\end{array}$ & $\begin{array}{c}\text { CEA } \\
\text { LVA } \\
\operatorname{EBCM}(16) \\
\operatorname{SVM}(16)\end{array}$ & $\begin{array}{c}1.24020 \mathrm{E}-02 \\
1.21981 \mathrm{E}-02 \\
- \\
1.20927 \mathrm{E}-02\end{array}$ & $\begin{array}{c}\text { 7.93953E-02 } \\
7.84549 \mathrm{E}-02 \\
- \\
7.80336 \mathrm{E}-02\end{array}$ \\
\hline 4 & $\begin{array}{c}a \\
b \\
a / b \\
V[i]\end{array}$ & $\begin{array}{c}1.00 \\
0.20 \\
0.60113\end{array}$ & $\begin{array}{c}0.80 \\
0.16 \\
5.00 \\
0.30778\end{array}$ & $\begin{array}{c}0.60 \\
0.12 \\
0.12984\end{array}$ & $\begin{array}{c}\text { CEA } \\
\text { LVA } \\
\text { EBCM }(16) \\
\text { SVM }(16)\end{array}$ & $\begin{array}{c}1.06311 \mathrm{E}-02 \\
1.04607 \mathrm{E}-02 \\
- \\
-\end{array}$ & $\begin{array}{c}\text { 7.26309E-02 } \\
7.18045 \mathrm{E}-02 \\
- \\
-\end{array}$ \\
\hline
\end{tabular}

Для частиц, рассматриваемых ниже, обе схемы вычисления поляризуемостей дают приближенные значения. Здесь эталонами выступают строгие методы ЕВСМ и SVM, области применимости которых, правда, не слишком широкие. Заметим, что в приведенных таблицах результаты, полученные методом SVM, предпочтительнее результатов, полученных методом ЕВСМ.

Численные расчеты сечений для трехслойных сфероидов с подобными слоями представлены в табл. 1.
Нетрудно увидеть, что в данном случае аппроксимация конфокальных сфероидов дает более точные результаты. Тем не менее отличие результатов двух приближенных формул составляет менее $2 \%$.

Табл. 2 содержит данные для трехслойных сплюснутых псевдосфероидов, поверхности слоев которых получаются в результате инверсии относительно начала координат подобных вытянутых сфероидов, которые имеют одинаковые отношения полуосей. Из нее видно, 
Таблица 3. То же, что в табл. 1, но для трехслойных подобных улиток Паскаля

\begin{tabular}{|c|c|c|c|c|c|c|c|}
\hline \multirow{2}{*}{ Случай } & \multirow{2}{*}{$\begin{array}{c}\text { Параметры } \\
\text { улитки Паскаля }\end{array}$} & \multicolumn{3}{|c|}{ № оболочки $(i)$} & \multirow{2}{*}{$\begin{array}{c}\text { Метод } \\
\text { расчета }\end{array}$} & \multirow{2}{*}{$\begin{array}{l}\text { Сечение } \\
\text { рассеяния }\end{array}$} & \multirow{2}{*}{$\begin{array}{c}\text { Сечение } \\
\text { поглощения }\end{array}$} \\
\hline & & 1 & 2 & 3 & & & \\
\hline \multirow[t]{2}{*}{1} & $\begin{array}{l}a \\
\epsilon\end{array}$ & 1.00 & $\begin{array}{c}0.80 \\
0.1\end{array}$ & 0.60 & $\begin{array}{l}\text { CEA } \\
\text { LVA }\end{array}$ & $\begin{array}{l}3.77285 \mathrm{E}-02 \\
3.70343 \mathrm{E}-02\end{array}$ & $\begin{array}{l}1.51144 \mathrm{E}-01 \\
1.48942 \mathrm{E}-01\end{array}$ \\
\hline & $V[i]$ & 1.01000 & 0.51712 & 0.21816 & $\begin{array}{l}\operatorname{EBCM}(16) \\
\operatorname{SVM}(16)\end{array}$ & $\begin{array}{l}3.77283 \mathrm{E}-02 \\
3.77283 \mathrm{E}-02\end{array}$ & $\begin{array}{l}1.51145 \mathrm{E}-01 \\
1.51145 \mathrm{E}-01\end{array}$ \\
\hline \multirow[t]{2}{*}{2} & $\begin{array}{l}a \\
\epsilon\end{array}$ & 1.00 & $\begin{array}{c}0.80 \\
0.3\end{array}$ & 0.60 & $\begin{array}{l}\text { CEA } \\
\text { LVA }\end{array}$ & $\begin{array}{l}4.31758 \mathrm{E}-02 \\
4.23851 \mathrm{E}-02\end{array}$ & $\begin{array}{l}1.60450 \mathrm{E}-01 \\
1.58141 \mathrm{E}-01\end{array}$ \\
\hline & $V[i]$ & 1.09000 & 0.55808 & 0.23544 & $\begin{array}{l}\operatorname{EBCM}(16) \\
\operatorname{SVM}(16)\end{array}$ & $\begin{array}{l}4.32647 \mathrm{E}-02 \\
4.32647 \mathrm{E}-02\end{array}$ & $\begin{array}{l}1.60788 \mathrm{E}-01 \\
1.60788 \mathrm{E}-01\end{array}$ \\
\hline \multirow[t]{2}{*}{3} & $\begin{array}{l}a \\
\epsilon\end{array}$ & 1.00 & $\begin{array}{c}0.80 \\
0.5\end{array}$ & 0.60 & $\begin{array}{l}\text { CEA } \\
\text { LVA }\end{array}$ & $\begin{array}{l}5.50138 \mathrm{E}-02 \\
5.40173 \mathrm{E}-02\end{array}$ & $\begin{array}{l}1.78633 \mathrm{E}-01 \\
1.76125 \mathrm{E}-01\end{array}$ \\
\hline & $V[i]$ & 1.25000 & 0.64000 & 0.27000 & $\begin{array}{l}\operatorname{EBCM}(16) \\
\operatorname{SVM}(16)\end{array}$ & $\begin{array}{c}5.597 \mathrm{E}-02 \\
5.57912 \mathrm{E}-02\end{array}$ & $\begin{array}{c}1.810 \mathrm{E}-01 \\
1.81158 \mathrm{E}-01\end{array}$ \\
\hline \multirow[t]{2}{*}{4} & $\begin{array}{l}a \\
\epsilon\end{array}$ & 1.00 & $\begin{array}{c}0.80 \\
0.7 \\
\end{array}$ & 0.60 & $\begin{array}{l}\text { CEA } \\
\text { LVA }\end{array}$ & $\begin{array}{l}7.50672 \mathrm{E}-02 \\
7.37327 \mathrm{E}-02\end{array}$ & $\begin{array}{l}2.05018 \mathrm{E}-01 \\
2.02245 \mathrm{E}-01\end{array}$ \\
\hline & $\begin{array}{l}V[i] \\
V[i]\end{array}$ & $\begin{array}{l}1.49000 \\
1.81000\end{array}$ & $\begin{array}{l}0.76288 \\
0.92672\end{array}$ & $\begin{array}{l}0.32184 \\
0.39096\end{array}$ & $\begin{array}{c}\operatorname{EBCM}(16) \\
\operatorname{SVM}(16) \\
\operatorname{EBCM}(16) \\
\operatorname{SVM}(16)\end{array}$ & $\begin{array}{c}- \\
7.82 \mathrm{E}-02 \\
- \\
-\end{array}$ & $\begin{array}{c}- \\
2.13 \mathrm{E}-01 \\
- \\
-\end{array}$ \\
\hline 5 & $\begin{array}{l}a \\
\epsilon\end{array}$ & 1.00 & $\begin{array}{c}0.80 \\
0.9\end{array}$ & 0.60 & $\begin{array}{l}\text { CEA } \\
\text { LVA }\end{array}$ & $\begin{array}{l}1.05954 \mathrm{E}-01 \\
1.04119 \mathrm{E}-01\end{array}$ & $\begin{array}{l}2.38903 \mathrm{E}-01 \\
2.35822 \mathrm{E}-01\end{array}$ \\
\hline
\end{tabular}

что здесь предпочтительной является аппроксимация LVA, относительные погрешности которой составляют величину, меньшую 2\%. Вторая аппроксимация СЕА отличается от первой снова менее, чем на $2 \%$.

Наконец, в табл. 3 представлены результаты для трехслойных улиток Паскаля с подобными слоями. В этом случае снова лучшей является аппроксимация СЕА, при этом ее относительные погрешности могут достигать 4\% (см. случай 4). Различия между двумя приближенными решениями, как и раньше, составляет не более $2 \%$.

Во всех трех таблицах последние случаи соответствуют частицам с параметрами, не удовлетворяющими условиям применимости строгих методов. В силу этого они дают заведомо неправильные результаты, которые не приводятся в таблицах. Отметим, что уменьшение количества значащих цифр для методов ЕBCM и SVM связано с увеличением вычислительных погрешностей при их применении. Здесь важно отметить, что в этих сложных случаях приближения CEA и LVA продолжают неплохо работать. О достоверности результатов приближенных решений свидетельствует их близость, а именно относительные погрешности меньше $2 \%$.

Эллипсоидальную модель совместно с приближением СЕА можно использовать для расчета „эффективной“ диэлектрической проницаемости слоистой несферической частицы. Для этого достаточно воспользоваться соотношением (19) для „эффективного“ многослойного эллипсоида.

\section{Заключение}

На основании выполненного аналитического и численного анализа эллипсоидальной модели для малых многослойных несферических частиц можно сделать следующие выводы.

1. Проведенное сравнение результатов численных расчетов по строгим и приближенным алгоритмам для конфокальных и неконфокальных слоистых сфероидов, трехслойных псевдосфероидов и улиток Паскаля показало, что относительные погрешности вычислений по приближенным формулам с использованием эллипсоидальной модели для сечений рассеяния и поглощения данных частиц составляют менее $2-4 \%$.

2. Приближение конфокального сфероида (CEA) предпочтительнее для слоистых сфероидов и улиток Паскаля, а линейная объемная аппроксимация (LVA), в рамках которой поляризуемость частицы представляется в виде линейной комбинации поляризуемостей „эффективных“ сфероидов пропорционально объемам слоев, лучше для слоистых псевдосфероидов. Различия между двумя приближенными решениями во всех случаях составляют менее $2 \%$. Следует отметить, что приближение СЕА нелинейно относительно объемов слоев частицы.

3. Область применимости эллипсоидальной модели для малых многослойных частиц значительно шире, чем таковая для строгих методов ЕBCM и SVM, использующих сферический базис для представления полей. 
О достоверности результатов приближенных решений свидетельствует их отличие друг от друга, не превышающее $2 \%$.

Работа была поддержана в 2017г. грантом ГУАП, грантом РФФИ № 16-02-00194а и грантом RFBR-DST № 16-52-45005.

\section{Список литературы}

[1] Борен К., Хафббмен Д. Поглощение и рассеяние света малыми частицами. М.: Мир, 1986.

[2] Kleinman R.E, Senior T.B.A. // Rayleigh scattering. In: Low and high frequency asymptotics. Ed. by V.K. Varadan, V.V. Varadan. Amsterdam: Elsevier, 1986. P. 1.

[3] Mishchenko M.I., Travis L.D., Lacis A.A. Scattering, Absorption and Emission of Light by Small Particles. Cambridge: Cambridge Univ. Press, 2002.

[4] Климов В.В. Наноплазмоника. М.: Физматлит, 2009.

[5] Морс Ф.М., Фешбах Г. Методы теоретической физики. М.: ИЛ, 1958.

[6] Lord Rayleigh // Phil. Mag. 1897. V. 44. P. 28.

[7] Moroz A. // J. Opt. Soc. Amer. B. 2009. V. 26. P. 517.

[8] Jones A.R. // Proc. Roy. Soc. London. 1979. V. A366. P. 111.

[9] Mackowski D.W. // Appl. Opt. 1995. V. 34. P. 3535.

[10] Klacka J., Kocifaj M. // J. Quant. Spectrosc. Rad. Transf. 2007. V. 106. P. 170.

[11] Li X., Min X., Liu D. // J. Opt. Soc. Am. A. 2014. V. 31. P. 1495.

[12] Kocifaj M., Klacka J., Videen G., Kohut I. // J. Quant. Spectrosc. Rad. Transf. 2012. V. 113. P. 2561.

[13] Фарафбонов В.Г. // Опт. и спектр. 2000. Т. 88. № 3. С. 441.

[14] Фараббонов В.Г. // Опт. и спектр. 2001. Т. 90. № 4. С. 646.

[15] Posselt B., Farafonov V.G., Ilin V.B., Prokopjeva M.S. // Meas. Sci. Technol. 2002. V. 13. P. 256.

[16] Sihvola A., Venermo J., Ylä-Oijala P. // Microwav. Tech. Lett. 2004. V. 41. P. 245.

[17] Ramm A.G. Wave Scattering by Small Bodies of Arbitrary Shapes. Singapore: World Scientific, 2005. 475 p.

[18] Gonzales F., Moreno F. // In: Light scattering from microstructures. Lectures Notes in Physics. Ed. by Moreno F., Gonzales F. Berlin: Springer, 2000. P. 1.

[19] Babenko V.A., Astafyeva L.G., Kuzmin V.N. Electromagnetic scattering by disperse media. London: Springer-Praxis, 2003. $432 \mathrm{p}$.

[20] Onaka T. // Ann. Tokyo Astron. Observ. 1980. V. 18. P. 1.

[21] Ciric I.R., Cooray F.R. // In: Light scattering by nonspherical particles. Ed. by Mishchenko M.I., Hovenier J.W., Travis L.D. San Diego: Academic Press, 2000. P. 89.

[22] Peterson B., Ström S. // Phys. Rev. D. 1974. V. 10. P. 2670.

[23] Wang D.S., Barber P.W. // Appl. Opt. 1979. V. 18. P. 1190.

[24] Mishchenko M.I., Videen G., Babenko V.A., Khlebtsov N.G., Wriedt T. // J. Quant. Spectrosc. Rad. Transf. 2004. V. 88. P. 357.

[25] Mishchenko M.I., Videen G., Babenko V.A., Khlebtsov N.G., Wriedt T. // J. Quant. Spectrosc. Rad. Transf. 2007. V. 106. P. 304.

[26] Al-Rizzo H.M., Tranquilla J.M. // J. Comp. Phys. 1995. V. 119. P. 356.

[27] Doicu A., Wriedt T., Eremin Y. Light scattering by systems of particles. Berlin: Springer, 2006. 371 p.
[28] Mishchenko, M.I., Hovenier J.W., Travis L.D. Light Scattering by Nonspherical Particles. San Diego: Academic Press, 2000. $690 \mathrm{p}$.

[29] Kahnert F.M. // J. Quant. Spectrosc. Rad. Transf. 2003. V. 79-80. P. 775.

[30] Фарабонов В.Г., Устимов В.И., Соколовская М.В. // Опт. и спектр. 2016. Т. 120. № 3. С. 470.

[31] Farafonov V., Ilin V., Ustimov V., Volkov E. // Adv. Math. Phys. 2017. V. 2017. Article ID 7862462. Р. 1.

[32] Фараббонов В.Г., Ильин В.Б. // Опт. и спектр. 2013. Т. 115. № 5. C. 836.

[33] Фарафонов В.Г., Ильин В.Б., Устимов В.И., Тулегенов А.Р. // Опт. и спектр. 2017. Т. 122. № 3. С. 506.

[34] Farafonov V.G., Il'in V.B. // J. Quant. Spectr. Rad. Trasf. 2014. V. 146. P. 244.

[35] Farafonov V.G., Ilin V.B., Ustimov V.I., Prokopjeva M.S. // J. Quant. Spectr. Rad. Trasf. 2016. V. 178. P. 176.

[36] Farafonov V.G., Sokolovskaya M.V. // J. Math. Sci. 2013. V. 194. P. 104. 